\title{
ASSISTENCIA DE ENFERMAGEM A PACIENTES COM MANIFESTAÇĀO DE COMPORTAMENTO DECORRENTE DE ALHEAMENTO DA REALIDADE
}

\author{
Marina Borges Teixeira* \\ Sónia Barros **
}

TEIXEIRA, M.B.; BARROS, S. Assistência de enfermagem a paciente com manifestação de comportamento decorrente de alheamento da realidade. Rev. Esc. Enf. $U S P$, v. 25 , n. 3, p. 335-46, dez. 1991.

Com este trabalho as autoras querem contribuir para que o enfermeiro tenha mais uma referência norteadora de sua ação ao assistir o paciente com manifestaçōes de alheamento da realidade. São descritos alguns conceitos de alheamento $e$, sumariamente, os quadros clinicos em que este se apresenta. Para a assistência de enfermagem são considerados os problemas do paciente $e$ as medidas gerais que devem ser utilizadas, levando-se em conta a individualidade do paciente.

UNITERMOS: Enfermagem psiquiátrica. Assistência de enfermagem.

\section{INTRODUÇĀO}

Como enfermeiras trabalhando em hospital psiquiátrico, ou como docentes de enfermagem psiquiátrica, acompanhando estudantes em suas atividades de campo, percebemos que tanto os membros da equipe de enfermagem como os estudantes têm dificuldades em atuar junto a pacientes com manifestações tipo alucinações, idéias delirantes, delírios, agressividade e agitação, entre outras. Estas dificuldades são evidenciadas, na literatura, por COSTA ${ }^{7}$, TEIXEIRA ${ }^{23}$, BURGESS ${ }^{6}$, TAYLOR ${ }^{22}$.

Tais manifestaçōes, que parecem dificultar a assistência de enfermagem, seja por causar medo ou por provocar ansiedade, são encontradas em diversos pacientes com quadro de perturbação mental inclusive aqueles com diagnóstico de esquizofrenia.

MERENESS ${ }^{14}$, KYES; HOFLING ${ }^{12}$, TRAVELBEE ${ }^{24}$, PEPLAU ${ }^{18}$, STUART, SUNDEENN ${ }^{21}$, BROWN, FOWLER 5, entre outros autores, ao

- Infermelra. Doutor em Enfermagem. Professor Assistente Doutor do Departamento de Infermagem Materno-Infantil e Psiquiátrica da Fscola de Fnfermagem da Universidade de São Paulo, disciplina Enfermagem Psiquítrica.

** Enfermeira Mestro em Enfermagem. Assistente do Departamento de Enfermagem Materno-Infantil e Paiquiátrica da Esscola de Enfermagem da Universidade de São Paulo, disciplina Enfermagem Psiquiátrica. 
abordarem a assistência de enfermagem a pacientes psiquiátricos, ressaltam a dificuldade da enfermeira ao assistí-los e, em particular, ao assistir àqueles cujo comportamento é caracterizado por alheamento da realidade.

O alheamento da realidade, segundo BROWN; FOWLER ${ }^{5}$, é isolar-se do meio ambiente. $O$ indivíduo parece absorvido em suas fantasias e exclui-se de qualquer interesse pela realidade.

Segundo TAYLOR ${ }^{22}$, alhear-se da realidade é a forma encontrada pelo doente para obter segurança pessual; o doente parece não ter capacidade para envolver-se emocionalmente e nem de forma duradoura com o seu ambiente.

No NURSING BOARDS REVIEW ${ }^{1}$ consta que a pessoa cujo comportamento é caracterizado por alheamento da realidade afasta-se de interações com os demais e do meio ambiente, afastando-se, portanto, da realidade.

O conceito de alheamento da realidade, elaborado pelas docentes da disciplina Enfermagem Psiquiátrica I da Escola de Enfermagem da USP é o seguinte: "Alheamento da realidade é um viver próprio, incompreensível para outras pessoas. Toda a vida do indivíduo está voltada para seu mundo interior, imaginário e impenetrável. O mundo exterior parece não ser percebido como real".

TRAVELBEE ${ }^{24}$, e MERENESS ${ }^{14}$ referem que pacientes cujo comportamento habitual é o isolamento têm, como diagnóstico médico mais comum, o de esquizofrenia.

Entendemos que um dos fatores que provocam ansiedade, medo e rejeição do paciente, gerando problemas para sua assistência, é decorrente da dificuldade de se entender o seu comportamento alheado, pois seu isolamento, indiferença afetiva ou empobrecimento emocional funcionam como verdadeiras barreiras que dificultam qualquer relaçăo efetiva e afetiva com este paciente. Ressaltamos, ainda, que o paciente não está alheado da realidade a todo momento e que mesmo a forma de alhear-se pode ser mais ou menos intensa, o que possibilita aos membros da equipe que o assiste oportunidade de atuarem terapeuticamente junto à ele.

Pretendemos, com este artigo, contribuir para que a enfermeira e o estudante de enfermagem disponham de mais uma fonte de informações a respeito da assistência de enfermagem a pacientes com manifestações de comportamento que caracterizam alheamento da realidade, decorrentes, especificamente, de quadros de esquizofrenia e, assim, possam desenvolver uma atitude de aceitação e compreensão em face do comportamento manifestado, atuando de forma terapêutica.

Inicialmente abordaremos de forma sucinta o conceito e as formas clínicas de esquizofrenia, para facilitar a compreensão da assistência a ser prestada. 
$\mathrm{Na}$ Classificação Internacional de Doenças Mentais (CID) ${ }^{15}$ encontramos os seguintes quadros esquizofrênicos:

- esquizofrenia tipo simples;

- esquizofrenia tipo hebefrênica;

- esquizofrenia tipo catatônica;

- esquizofrenia tipo paranide;

- episódio esquizofrênico agudo;

- esquizofrenia latente;

- esquizofrenia residual;

- tipo esquizo-afetivo;

- outras formas.

No presente estudo deter-nos-emos nos quadros de esquizofrenia tipo simples, hebefrênico e catatônico, por serem bastante característicos e seus sintomas amplamente estudados e descritos na literatura.

BLEULER ${ }^{4}$, descrevendo o quadro psicopatológico da esquizofrenia, dá ênfase às alterações do curso do pensamento e da afetividade e a alterações na vivência subjetiva da própria personalidade. Relacionados a estas alterações, as áreas da vontade e da atividade também estão alteradas. O autor chama a atenção para o fato de que ambivalência e o autismo constituem aspectos importantes da esquizofrenia; ressalta, ainda, que a percepção, orientação, memória e atenção são conservadas, sofrendo alterações indiretas das alterações dos sintomas básicos da esquizofrenia.

Kurt Schneider, citado por VILELA ${ }^{24}$, descreve uma série de sintomas da esquizofrenia, aos quais denomina sintomas de primeira ordem, cuja presença, excluída causa orgânica, seria indicativa de um quadro esquizofrênico. São eles: "sonorização do pensamento, escutar vozes que dialogam entre si, escutar vozes que acompanham a própria ação, vivência de influência corporal, roubo de pensamento, percepção delirante e vivência de influência no domínio dos sentimentos, tendências e vontade".

EY et $\mathrm{al}^{8}$ referem que a esquizofrenia pode ser entendida como um transtorno no qual predominam distúrbios psicopatológicos como incoerência entre pensamento e verbalização, ambivalência ideativa e volitiva, idéias delirantes, alucinações e perturbações afetivas assim como autismo.

Autismo, segundo PAIM ${ }^{16}$, é manifestação comum a todas as formas de esquizofrenia. Não significa o afastamento do indivíduo da realidade, mas representa incapacidade do paciente para estabelecer relações "normais" com o ambiente.

Descreveremos a seguir as três (3) formas de esquizofrenia que, a nosso ver, ilustram melhor o quadro clínico que caracteriza o comporta- 
mento alheado da realidade; baseamo-nos nos autores EY et all ${ }^{8}$, SOLOMON, PATCH ${ }^{19}$, PAIM ${ }^{16}$, KAPLAN; SADOCK ${ }^{10}$, KOLB ${ }^{11}$ e ATHAYDE ${ }^{3}$, para elaborar o resumo que segue.

\section{ESQUIZOFRENIA TIPO SIMPLES}

Neste quadro predominam o empobrecimento aparente do pensamento e das manifestações afetivas. O paciente isola-se do seu círculo familiar e de amizades, mostra desinteresse geral por tudo e por si mesmo. Esta indiferença é crescente dando a impressão de que 0 paciente apresenta algum tipo de déficit intelectual.

Ele pode apresentar distúrbios de sensopercepção (alucinação) e de pensamento (idéias delirantes), porém esta sintomatologia é, no geral, minima e sem persistência.

o quadro ocorre mais comumente em pessoas jovens que, até o momento de sua eclosão, tiveram evolução psíquica normal. A impressão causada é de que quando a doença se iniciou, ocorreu uma parada nessa evolução e a pessoa entrou em um quadro regressivo, com rápida desestruturação da personalidade.

\section{ESQUIZOFRENIA HEBEFRENICA}

É o quadro no qual a sintomatologia manifesta-se de modo mais exuberante.

O comportamento do paciente é pueril e bizarro; canta, pula, dança, corre, ri e chora e, às vezes, chega a andar como se fosse um animal; apresenta várias idéias delirantes, porém não estruturadas ou sistematizadas. Ocorre perda gradual do contacto com a realidade.

O paciente pode apresentar, também, verborréia, linguagem rebuscada e comportamento teatral, pode tornar-se agressivo consigo mesmo, com outras pessoas e com objetos. Se não for tratado, isola-se cada vez mais em seu mundo interior, tornando sua forma de agir e de relacionar-se com o ambiente incompreensivel para os demais.

\section{ESQUIZOFRENIA TIPO CATATÓNICA}

Neste quadro predominam alterações nas áreas de vontade e atividade. O paciente apresenta uma redução da mímica facial e corporal; no geral não responde verbalmente às solicitações que lhe são feitas, não deixa transparecer que as entendem ou, mesmo, que as ouviu.

Às vezes faz o contrário do que lhe é solicitado, outras não se movimenta nem para atender a suas necessidades fisiológicas; é possível que apresente também "flexibilidade ceréa", catalepsia, passividade, impulsões, podendo chegar a um quadro de intensa agitação e agressividade chamado de "furor catatônico". 


\section{ASSISTENCIA DE ENFERMAGEM}

Para a descrição da assistência de enfermagem a pacientes com comportamento caracterizado por alheamento da realidade fundamentamo-nos nas obras de MERENESS ${ }^{14}$, MATHENEY; TOPALIS ${ }^{13}$, STUART; SUNDEEN ${ }^{21}$, BURGESS ${ }^{6}$, KYES; HOFLING ${ }^{12}$, SHIVES ${ }^{18}$, IRVING ${ }^{9}$, STEFANELLI; ARANTES ${ }^{20}$ e WILSON; KNEISL ${ }^{25}$.

Descreveremos esta assistência geral de enfermagem com ênfase na prestada a pessoas hospitalizadas, e ressaltamos que deverá ser considerada a individualidade de cada paciente.

O primeiro passo para a assistência de enfermagem é a observação e descrição do comportamento do paciente. Para tanto, recomendamos que seja seguido o roteiro elaborado por ARANTES ${ }^{2}$. Os dados desta observação serão completados com informações obtidas em outras fontes, tais como o prontuário do paciente, seus familiares e os membros da equipe multiprofissional.

Essas informações permitirão à enfermeira identificar os problemas sentidos pelo paciente, decorrentes ou não de sua sintomatologia, bem como os problemas que podem surgir para a assistência de enfermagem.

\section{PROBLEMMAS PARA O PACIENTE}

- não se considera doente, não aceitando a internação e o tratamento;

- sente-se ansioso quando percebe os sintomas da doença, principalmente no início de suas manifestações;

- sente-se incompreendido e rejeitado e não entende o porque disto, isolando-se cada vez mais;

- sofre quando os outros demonstram ter medo dele e não compreende o motivo desse medo.

\section{PROBLEMAS PARA ASSISTENCIA DE ENFERMAGEM}

Em face das manifestações de comportamento do paciente os membros da equipe de enfermagem podem encontrar problemas para assistí-lo. Os principais são:

- dificuldade em compreender que as manifestações de comportamento do paciente são decorrentes de sua doença;

- frustração pela falta de ressonância, que às vezes existe aos estímulos exteriores e aos esforços para ajudá-lo;

- falta de compreensão da comunicação verbal e não verbal do paciente, o que dificulta a identificação de seus problemas, gera irritação, ansiedade e tendência a isolá-lo cada vez mais ou, ao contrário, a envolver-se de forma não terapêutica com ele;

- dificuldade em identificar sintomas, principalmente quanto está em mutismo ou cria palavras novas; 
- dificuldade em mantê-lo na realidade;

- dificuldade em protegê-lo e aos demais, quando manifesta auto ou hetero agressividade;

- dificuldade em conseguir que cuide de sua aparência, higiene, alimentação;

- dificuldade em evitar que seja alvo de zombaria dos demais pacientes ou por estes maltratado;

- dificuldade em prevenir complicações somáticas.

$O$ objetivo geral da assistência de enfermagem a paciente com manifestações de comportamento decorrentes de sintomas de alheamento da realidade é: trazê-lo e mantê-lo na realidade.

Os objetivos específicos serão traçados de acordo com as manifestações de comportamento de cada paciente em particular.

\section{MEDIDAS GERAIS DE ASSISTENCIA DE ENFERMAGEM A SEREM TOMADAS PELA ENFERMEIRA}

\section{- OFERECER APOIO.}

Oferecer apoio, permanecendo ao lado do paciente nos momentos difíceis, tais como quando este apresenta sinal de medo decorrente das alucinações e das idéias delirantes, evitando julgá-lo ou condená-lo pelo seu comportamento e demonstrando empatia, interesse e disponibilidade em ajudá-lo.

Ajudá-lo a exprimir seus pensamentos e sentimentos, esforçando-se para entender o que ele está tentando transmitir.

Para tanto, valer-se das técnicas de comunicação terapêutica, entre elas, saber ouvir, usar terapeuticamente o silêncio, pedir que esclareça o que não foi entendido, que são algumas das mais utilizadas; demonstrar interesse e aceitá-lo, verbalizando esta aceitação, é imprescindível; estar atenta para evitar o uso de técnicas de comunicação terapêutica que possam trazer maiores problemas para o paciente, como, por exemplo, usar frases reticentes ou em aberto com aqueles que apresentam desagregação do pensamento.

Estar atenta para sua própria comunicação não verbal - postura, gestos e expressão facial - que deve ser congruente com a verbal e transmitir interesse e disposição em ajudar o paciente.

Mantê-lo na realidade a maior parte do tempo possivel.

Quando o paciente apresentar alucinações, chamá-lo pelo nome, conversar sobre fatos que estejam ocorrendo ao seu redor, sugerindo atividades que captem sua atenção. Se o paciente insistir em falar sobre as alucinações, permitir que o faça por alguns momentos e, em seguida, procurar voltar sua atenção para fatos ou atividades concretas.

Quando os pacientes expressam idéias delirantes, estas não devem ser discutidas, mas sim ouvidas com atenção, por alguns instantes, e, gradativamente, levar o paciente a voltar sua atenção para fatos ou atividades concretas. 
As atividades individuais ou glupais contribuem para a remissão destes sintomas. Estas devem ser simples, manuais, variadas, dentro das possibilidades de cada paciente.

Em ambos os casos, de alucinações e idéias delirantes, deixar bem claro ao paciente que esta experiência é só dele, que acredita no que ele está vivenciando porém, que ela, enfermeira, não tem a mesma vivência.

A expressão de alucinações e idéias delirantes por parte do paciente pode provocar desconforto e ansiedade na enfermeira, que precisa ser capaz de controlá-la.

Não usar os sintomas apresentados pelo paciente pará obter sua cooperação.

Por exemplo, no caso de negativismo o paciente pode não fazer aquilo que lhe é solicitado ou fazer exatamente o contrário.

Às vezes, os sintomas são tão intensos que o paciente chega a negar sua necessidade de alimentar-se, higienizar-se e na maioria das vezes, nega-se a participar de qualquer atividade. Neste caso, a enfermeira, deve empenhar-se em conseguir a confiança do paciente, demonstrando interesse e disponibilidade de tempo para ajudá-lo, e dar-lhe tempo para tomar iniciativa quanto ao que fazer. Condutas como pedir ao paciente que faça exatamente o contrário do que a enfermeira deseja só reforçam e agravam o quadro.

O incentivo para participar no tratamento, nas atividades de vida diária e no autocuidado deve ser feito com frequencia, para acelerar seu processo de recuperação.

\section{PROTEGER O PACIENTE E AOS DEMAIS QUANDO ESTE} APRESENTAR AGRESSIVIDADE

Quando o paciente apresentar manifestação de comportamento que indiquem agressividade, analisar a situação e tomar as medidas cabiveis.

Limitar o ambiente, diminuindo estímulos, falar em tom de voz baixo, porém firme, identificar fonte de irritação para o paciente e ocupá-lo são algumas dessas medidas. Quando a agressividade não puder ser controlada desta forma, faz-se necessário isolar o paciente em um ambiente sem estímulos externos - uma sala por exemplo - onde possa estar junto com um funcionário e onde terá oportunidade de se recuperar. O paciente não deve sentir, em momento algum, que esta medida foi adotada como castigo.

Em casos extremos, quando os recursos citados foram adotados sem sucesso, isolar o paciente em seu quarto e, por vezes, contê-lo física e quimicamente. A enfermeira adotará esta medida após avaliação cuidadosa da situação e avisará o médico sobre a conduta tomada, pedindolhe que também avalie o paciente. Este deverá ser orientado do porquê de tal conduta, mesmo que dê mostra de não estar compreendendo, no momento. 
A enfermeira deve, também, estar atenta quanto aos cuidados especificos nos casos de isolamento e contensão.

\section{EVITAR SITUAÇŐES DE CONFLITO QUE GEREM ANSIEDADE NO PACIENTE, QUANDO ESTE ESTIVER AMBIVALENTE}

Evitar situações nas quais o paciente tenha que tomar decisão; quando necessário, decidir por ele até que este readquira essa capacidade.

\section{EVITAR FUGA, SUICIDIO E AUTOMUTILAÇĀO}

Criar e manter um ambiente terapêutico que proporcione ao paciente segurança e o atendimento de suas necessidades; usar as medidas indicadas para impedir estes problemas e saber prever quando os mesmos vão surgir. Manter o paciente ocupado em atividades é uma das medidas mais eficazes para estes casos.

CONSEGUIR QUE O PACIENTE CUIDE DE SUA HIGIENE, APARENCIA E QUE ACEITE AJUDA E ORIENTAÇÃO

Em geral, a atenção do paciente está voltada para seus pensamentos e ele descuida-se dos aspectos acima citados. Neste caso, ficar a seu lado estimulando-o e elogiá-lo quando começar a se cuidar, fazendo por ele aquilo que não for capaz.

Observar e orientar quanto ao uso adequado de vestes. Por exemplo, se está de acordo com o clima.

\section{OBSERVAR FUNÇŌES FISIOLÓGICAS E CONDIÇŌES FISICAS, INDAGANDO DO PACIENTE SOBRE AS MESMAS}

O paciente deve ser orientado sobre o motivo destas indagações e a importância de sua cooperação. Nem sempre o paciente encontra-se em condições de responder; a enfermeira e os membros da equipe de enfermagem devem, neste caso, observar cuidadosamente este fato. Se for constatado algum problema, tomar as medidas cabiveis, como avisar o médico e providenciar dieta apropriada, entre outras.

\section{VERIFICAR ALIMENTAÇÃO E HIDRATAÇÃO}

Estar presente no horário das refeições para verificar se há problemas decorrentes ou não da sintomatologia; identificar as causas do problema e procurar saná-las. Nem sempre o paciente lembra-se de ingerir líquidos; porisso compete à enfermeira deixar água em recipientes adequados e em local de fácil acesso, para os pacientes dela se servirem ou ela própria oferecer-lhes periodicamente.

\section{OBSERVAR SONO}

Observar se o paciente está dormindo à noite. Às vezes, este permanece deitado, sem dormir. E importante conhecer seus hábitos de sono antes de ir para a cama, saber se durante o dia participou de atividades 
ou permaneceu deitado. Uma vez identificada como problema a falta de sono, tomar medidas que auxiliem o sono tais como: banho morno, beber leite, permitir que fique lendo até ter sono, conversar com o paciente ocupá-lo durante o dia, entre outras. Caso todas falhem, comunicar ao médico para prescrição de medicação.

\section{FAZER COM QUE O PACIENTE ACEITE A INTERNAÇÃO E TRATAMENTO}

Isto nem sempre é fácil de se conseguir, particularmente com os pacientes que não se julgam doentes. Se for este o caso, oferecer apoio, demonstrando compreensão para com os sentimentos do paciente, porém sendo sempre sincera, não usando de artifícios tais como: "o Sr. só vai ficar aqui para fazer exame" ou "sua familia acha que o Sr. deve ficar internado"; tentar conseguir a confiança do paciente e levá-lo a perceleite, permitir que fique lendo até ter sono, conversar com o paciente, ber a necessidade da internação. Não se considerando doente, o paciente recusa o tratamento. A mesma medida deve ser tomada; levá-lo a compreender a importância do mesmo, ressaltando os aspectos positivos do tratamento e orientando o paciente, sobre sua necessidade. Caso este tome medicação oral, observar se a está ingerindo, não mentir nem esconder medicações nos alimentos. Permanecer atenta aos efeitos colaterais do tratamento, orientando o paciente a respeito $\mathrm{e}$ tomando as precauções e cuidados cabíveis.

As mesmas medidas devem ser tomadas em relação a outros tipos de tratamento: ECT, insulinoterapia.

\section{ESTABELECER LIMITES}

Estes devem ser impostos pela enfermeira quando o paciente apresentar manifestações de comportamento anti-social tais como despir-se em público, usar termos de baixo calão, provocar os demais, não seguir as normas da instituição, tentar manipular os demais ou agredir. Os limites devem ser impostos de forma clara para o paciente e mantidos por todos que o atendem; e têm como objetivo mudar o comportamento não recomendável para outro, socialmente aceitável.

Os limites também devem ser impostos com a mesma firmeza aos demais pacientes, quando provocarem ou transformarem 0 paciente em alvo de chacota, no caso deste, apresenta o comportamento bizarro característico do quadro de esquizofrenia e hebefrênica.

\section{SOCIALIZA-LO GRADATIVAMENTE}

Após analisar as condições do paciente, procurar socializá-lo acompanhando seu ritmo. Dependendo do paciente podem ser indicados, no inicio, atividades em pequenos grupos, que, aos poucos podem ser ampliados. 


\section{AJUDAR O PACIENTE A SE COMUNICAR}

Uma grande dificuldade do paciente é na comunicação com os demais. Usar, com ele, linguagem clara, simples, frases curtas, não elaboradas que lhe permitam compreender a mensagem e responder as perguntas feitas. Sempre que a mensagem do paciente não for compreendida, pedir esclarecimentos. $O$ uso de técnicas de comunicação terapêutica deve ser planejado para cada paciente em particular, levados em conta suas dificuldades. Atenção constante à comunicação nãoverbal do paciente, que com freqüência fornece informações importantes sobre seus sentimentos.

\section{ANALISAR PLANOS PARA O FUTURO}

À medida em que a enfermeira e paciente vão se conhecendo e atuando juntos, os projetos futuros do paciente emergem. Compete à enfermeira, ajudá-lo a analisar estes planos, verificando juntos as possibilidades ou não de concretizá-los. Esta análise, porém, só deve ser feita quando o paciente tiver condição para tal. E importante ressaltar que o paciente é quem deve fazê-la. A enfermeira deverá ajudá-lo, verificando se os planos estão ou não de acordo com a realidade, discutindo e levando o paciente a tomar ciência das vantagens e desvantagens do planejado, estimulando-o quando seus projetos forem viáveis.

\section{ORIENTAR SOBRE LICENÇA E ALTA MÉDICA}

É necessário orientar o paciente sobre a licença autorizada pelo médico, a necessidade do retorno e a importância de continuar com o tratamento. Às vezes, ele pode mostrar-se inseguro com a sua saída do hospital seja de alta ou licença. Orientá-lo quando ao retorno ao hospital nas datas marcadas e possibilidade de ser atendido independentemente das mesmas; reforçar a importância do retorno ao ambulatório e da continuidade do tratamento.

Dar aos membros da família do paciente a mesma orientação, e verificar quais suas dúvidas e seus temores, esclarecendo-os.

\section{ANOTAR OS CUIDADOS PRESTADOS}

Anotar na papeleta do paciente toda e qualquer assistência que lhe for prestada. A anotação deve ser a mais completa possível e incluir: breve descrição do comportamento do paciente, idéias que expressa, atividades exercidas e não exercidas, funções fisiológicas, alimentação, aparēncia, sono, queixas, intercorrências e tratamento. Além do aspecto legal, a anotação de enfermagem fornece elementos para verificação da eficácia ou não do tratamento, bem como da evolução do paciente.

Nem sempre a enfermeira é quem se encarregará das anotações; outros membros da equipe de enfermagem devidamente orientados, o 
farão. Rcomenda-se, porém, que pelo menos uma vez por semana, a enfermeira avalie o paciente e faça sua anotação.

\section{CONTROLAR OS PRÓPRIOS SENTIMENTOS}

Como já foi dito anteriormente, o paciente com comportamento alheado da realidade, pode, pelas próprias caracteristicas de sua doença, gerar ansiedade, irritação, frustração, medo, insegurança e outros sentimentos na enfermeira. Esta, deve ser capaz de controlá-los a fim de evitar que os mesmos prejudiquem a assistência a ser prestada.

Deve reunir-se com os demais membros da equipe de enfermagem, discutir os problemas e encontrar soluçōes para os mesmos em conjunto; estimular seus funcionários a falarem abertamente sobre seus sentimentos em relação ao paciente e identificar, no grupo, quais as pessoas mais indicadas para ficarem ao lado do paciente e quais aquelas cujo tempo ao lado do mesmo deve ser reduzido.

\section{CONSIDERAÇÕES FINAIS}

O comportamento do paciente alheado da realidade parece provocar, na equipe de enfermagem, sentimentos de medo e rejeição. O planejamento da assistência de enfermagem, deve considerar os problemas que este comportamento traz tanto para o paciente como para os membros da equipe de enfermagem, e prever as medidas necessárias para amenizá-los. Ressaltamos que estas medidas, em si, isoladamente, não ajudarão os pacientes se não for levada em conta a individualidade de cada um e, principalmente, a sua participação, dentro de suas possibilidades, no planejamento e na implementação do plano para a sua recuperação.

TEIXEIRA, M.B.; BARROS, S. Nursing care of patients with behavioural manifestation due to reality withdrawal. Rev. Esc. Enf. USP, v. 25, n. 3, p. 335-46, Dec. 1991.

With this study the autors want to furnish the nurses with one more reference source to guide their actions in caring for the patient with manifestation of reality withdrawal.

Some concepts of withdrawal are presented and, summarely, the clinical manifestation. Nursing care is described considering their problems and general measures to ie taken into account for patients individuality.

UNITERMS: Psychiatric nursing. Nursing care.

\section{REFERENCIAS BIBLIOGRAFICAS}

AMERICAN JOURNAL OF NURSING. Nursing boards review. Paclfic Palisades, Nurseco, 1985. p. 73-83.

ARANTES, E.C. Observação de comportamento de pacientes internados em hospital psiquítrico. Rov. Bras. Enf., v. 21, n. 1/3, p. 39-49, 1968.

ATHAYDE, J.S. de. Flementos de psicopatologia. 3. ed. Lisboa, Fundaç̃o Calouste Gulbenbrian, 1979. cap. 8, p. 282-94: As psicoses. 
BLEULER, E. Psiquiatria. 15. ed. Rio de Janeiro, Guanabara, Koogan, 1985.

BROW, M.M.; FOWLER, G.R. Psychodinamic nursing: a biosocial orientation. 4. ed. Philadelphia, Saunders, 1971. cap. 14, p. 197-207: the patient with autistic behavior.

BURGeSS, A.W. Psychiatric nursing in the hospital and community. 4. ed. EnglewoodCliffs, Prentice-Hall, 1985. cap. 13, p. 274-316: Schizophrenia and paranoid dysfunctional patherns.

COSTA, A.E. Características do paciente psiquiátrico considerado problema pelo pessoal de enfermagem. São Paulo, 1977, p. 124. Dissertação (Mestrado) - Escola de Enfermagem, Universidade de são Paulo.

EY, H. et al. Manual de psiquiatria. 5. ed. Rio de Janeiro, Masson do Brasil, 1981. cap. 13, p. 534-615: Psicoses esquizofrênicas.

IRVING, S. Enfermagem pgiquítrica basica. 2. ed. Rio de Janeiro, Interamericana, 1978. cap. 10, p. 119-43: Reações esquizofrênicas ao stress.

KAPLAN, H.I.; SADOCK, B.J. Compêndio de psiquiatria dinâmica. 3. ed. Porto Alegre, Artes Médicas, 1984 . cap. 13, p. 260-303: Perturbações esquizofrenicas.

KOLB, L.C. Psiquiatria clínica. 9. ed. Rio de Janeiro, Interamericana, 1977. cap. 19, p. 287-332: Psicoses esquizofrênicas.

KYES, J.J.; HOFLING, C.M. Conceitos básicos em enfermagem psiquiátrica. 4. ed. Rio de Janeiro, Interamericana, 1985. cap. 14. p. 260-78: As psicoses: esquizofrenia e estados paranóides.

MATHENEY, R.V.; TOPALIS, M. Enfermería psiquiatrica. 3. ed. México, Interamericana, 1962. cap. 8, p. 111-25: Cuidados de los retraidos.

MERENESS, D. Elementos de enfermería psiquiátrica. 2. ed. Méxíco, Prensa Médica Mexicana, 1973. cap. 10, p. 129-51: Pacientes cuya conducta se caracteriza por tendencias al aislamiento.

ORGANIZACAO MUNDIAL DA SAUDE. Classificação internacional das doenças mentais: CID-9. Washington, 1978.

PAIM, I. ERquibofrenia. 2. ed. São Faulo, Grijalbo, 1973. p. 31-155.

PHPLAU, H. Prinofios básicos para la orientación del paciente. Washington, OPAS/OMS, 1968.

SHIVES, L.R. Basic concepts of psychiatric mental health nursing. Philedelphia, Lippincott, 1983. cap. 25, p. 485-502: Imparred thought process schizofrenic desorders.

SOLOMON, P.; PATCH, U. Manual de psiquiatria. São Paulo, Atheneu/EDUSP, 1975. cap. 13, p. 179-97: As esquizofrenias.

STEFANELIC, M.C.; ARANTES, E.C. Descrição das manifestações de comportamento apresentadas por pacientes com diagnóstico de esquizofrenia. Rev. Bras. Enf., v. 28, n. 4, p. 9-21, 1975.

STUART, G.W.; SUNDEEN, S.J. Principles and practice of psychiatric nuraing, 2. ed. Saint Louis, Mosby, 1988 . cap. 11, p. 358-401: Disruption in relatedness.

TAYLOR, C.M. Mereness' essentials of psychiatric nursing. 12. ed. Saint Louis, Mosby, 1982. cap. 13, p. 271-99: Behavioral patherns that reflect maladaphalive mood state.

TEIXEIRA, M.B. Manifestacōes do doente mental não toleradas pelo pessoal de enfermagem. São Paulo, 1978. Dissertação (Mestrado) - Escola de Enfermagem, Universidade de São Peulo.

TRAVELBEE, J. Intervencion en enfermeria psiquiátrica. Colombla, Carvajal, 1979. cap. 9, p. 160-71: Areas criticas en la relación enfermera-paciente.

VILFHA, W.A. Os sintomas de primeira ordem (SPO) de Kurt Schneider na esquizofrenia: relação com o curso evolutivo e a personalidade. J. Bras. Psiq., v. 38, n. 4, p. 165-73, 1989.

WILsON, H.S.; KNEISL, C.R. Psychiatric nursing. Menlo Park, Addison-Wesley, 1979. cap. 13, p. 340-88: Desintegrative patterns.

Recebido em 8-11-90

Aprovado em 14-3-91 